\title{
Inflection point in the power spectrum of stellar brightness variations
}

\section{Facular versus spot dominance on stars with known rotation periods ${ }^{\star}$}

\author{
E. M. Amazo-Gómez ${ }^{1,2}$, A. I. Shapiro ${ }^{1}$, S. K. Solanki ${ }^{1,3}$, G. Kopp ${ }^{4}$, M. Oshagh ${ }^{2,5}$, T. Reinhold ${ }^{1}$, and A. Reiners ${ }^{2}$ \\ ${ }^{1}$ Max-Planck-Institut für Sonnensystemforschung, Justus-vonustus-von-Liebig-Weg 3, 37077 Göttingen, Germany \\ e-mail: amazo@mps.mpg.de \\ ${ }^{2}$ Georg-August Universität Göttingen, Institut für Astrophysik, Friedrich-Hund-Platz 1, 37077 Göttingen, Germany \\ ${ }^{3}$ School of Space Research, Kyung Hee University, 446-701 Yongin, Gyeonggi, Korea \\ ${ }^{4}$ Laboratory for Atmospheric and Space Physics, 3665 Discovery Dr., Boulder, CO 80303, USA \\ ${ }^{5}$ Instituto de Astrofísica de Canarias (IAC), 38200 La Laguna, Tenerife, Spain
}

Received 14 July 2020 / Accepted 26 August 2020

\begin{abstract}
Context. Stellar rotation periods can be determined by observing brightness variations caused by active magnetic regions transiting visible stellar disk as the star rotates. Successful stellar photometric surveys stemming from the Kepler and TESS observations have led to the determination of rotation periods in tens of thousands of young and active stars. However, there is still a lack of information on the rotation periods of older and less active stars like the Sun. The irregular temporal profiles of light curves caused by the decay times of active regions, which are comparable to, or even shorter than, stellar rotation periods, in combination with the random emergence of active regions make period determination for such stars very difficult.

Aims. We tested the performance of a new method for the determination of stellar rotation periods against stars with previously determined rotation periods. The method is based on calculating the gradient of the power spectrum (GPS) and identifying the position of the inflection point (i.e. point with the highest gradient). The GPS method is specifically aimed at determining rotation periods of low-activity stars like the Sun.

Methods. We applied the GPS method to 1047 Sun-like stars observed by the Kepler telescope. We considered two stellar samples individually: one with near-solar rotation periods (24-27.4 d) and a broad range of effective temperatures (5000-6000 K) and the other with near-solar effective temperatures $(5700-5900 \mathrm{~K})$ and a broad range of rotation periods (15-40 d).

Results. We show that the GPS method returns precise values for stellar rotation periods. Furthermore, it allows us to constrain the ratio between facular and spot areas of active regions at the moment of their emergence. We also show that the relative facular area decreases with the stellar rotation rate.

Conclusions. Our results suggest that the GPS method can be successfully applied to retrieve the periods of stars with both regular and non-regular light curves.
\end{abstract}

Key words. stars: rotation - stars: solar-type - methods: observational - techniques: photometric

\section{Introduction}

Rotation periods in cool main-sequence stars can be traced by observing the brightness modulation caused by the presence of active regions on stellar surfaces. Those active regions are generated by the emergence of strong localized magnetic fields approximately described by flux tubes (see e.g. Solanki 1993). Large flux tubes form dark spots, while ensembles of smaller flux tubes form bright faculae (see, e.g. Solanki et al. 2006, for a detailed review of the solar case). The active regions usually consist of a sunspot group surrounded by faculae. The transits of such active regions over the visible disk as the star rotates would cause brightness variability. Consequently, the stellar light curves (LCs) contain information about both the rotation periods and the properties of active regions. However, retrieving this information from the LCs often proves a daunting task (see e.g. Basri 2018).

${ }^{\star}$ Full Table 2 is only available at the CDS via anonymous ftp to cdsarc.u-strasbg.fr $(130.79 .128 .5)$ or via http://cdsarc. u-strasbg.fr/viz-bin/cat/J/A+A/642/A225
The Kepler mission (Borucki et al. 2010) has provided the community with records of photometric observations with unprecedented precision and cadence. The Kepler LCs have been widely used to determine stellar rotation periods (e.g. Walkowicz \& Basri 2013; Reinhold \& Gizon 2015; Nielsen et al. 2013; García et al. 2014; McQuillan et al. 2014; Buzasi et al. 2016; Angus et al. 2018; Santos et al. 2019). Despite considerable success in determining the rotation periods of many fast-rotating and active stars (see, e.g. McQuillan et al. 2014, who published rotation periods of about 34030 stars identified as being located on the main sequence) there is a lack information on periods of slowly rotating stars, that is, stars with near-solar and longer rotation periods. For example, the rotational period of the Sun may not be detectable during intermediate and high levels of solar activity (see Lanza \& Shkolnik 2014; Aigrain et al. 2015).

The difficulties in detecting periods of slowly rotating stars might be an important contribution to the explanation of lowerthan-expected numbers of G-type stars with near-solar rotation periods (van Saders et al. 2019). The difficulty in obtaining a reliable measurement of the rotation periods of stars with variability 
Table 1. Stellar parameters for samples A and B.

\begin{tabular}{lcccccc}
\hline \hline Sample & $\mathrm{N}$ & $T_{\mathrm{eff}}{ }^{(1)}(\mathrm{K})$ & $\log g^{(1)}$ & {$[\mathrm{Fe} / \mathrm{H}]^{(1)}$} & $\operatorname{Var}^{(2)}(\mathrm{ppm})$ & $P_{\text {rot }}{ }^{(2)}(\mathrm{d})$ \\
\hline A & 686 & $5000-6000$ & $4.20-4.69$ & $-1.46-0.56$ & $211-39748$ & $24.0-27.4$ \\
B & 361 & $5700-5900$ & $4.21-4.60$ & $-1.08-0.44$ & $211-17530$ & $15.0-39.8$ \\
Sun & 1 & 5778 & 4.44 & 0.0 & $300-1500$ & $27.27(\mathrm{Sy}), 25.38(\mathrm{Sid})$ \\
\hline
\end{tabular}

Notes. Stellar parameters for stellar samples A and B. ${ }^{(1)}$ Effective temperature $\left(T_{\text {eff }}\right)$, surface gravity $(\log g)$, and metallicity $([\mathrm{Fe} / \mathrm{H}])$ values are taken from Huber et al. (2014). ${ }^{(2)}$ Variability range (Var) and rotation periods $\left(P_{\text {rot }}\right)$ are taken from McQuillan et al. (2014). We take the solar synodic (Sy) and sidereal (Sid) Carrington rotation period values as a reference.

patterns similar to that of the Sun can also affect solar-stellar comparison studies (see e.g. Witzke et al. 2020; Reinhold et al. 2020).

Within this context, we have developed a method aimed at determining rotation periods of low-activity stars like the Sun. In Shapiro et al. (2020) (hereinafter, Paper I), we found that the power spectra of brightness variations of such stars are strongly affected by the evolution of active regions. In particular, the rotation peak can be significantly weakened or it may even disappear from the power spectrum if the lifetimes of starspots are too short. Furthermore, the delicate balance between spot and facular contributions to the variability might lead to the appearance of spurious peaks, which do not correspond to the rotation period but could be easily mistaken for one (see also Shapiro et al. 2017).

In Paper I, we showed that the high-frequency tail of the power spectrum is much less affected by the evolution of magnetic features than frequencies near the rotation period. Consequently, we proposed to use information in the high-frequency tail for the determination of stellar rotation periods. In particular, we suggested that the period, $P_{\mathrm{HFIP}}$, corresponding to the maximum of the gradient of the power spectrum (GPS) (i.e. to the inflection point) in the high-frequency tail could be used to identify the stellar rotation period, $P_{\text {rot }}$, via the simple scaling relation:

$$
P_{\text {rot }}=P_{\mathrm{HFIP}} / \alpha \text {. }
$$

Here, $\alpha$ is a calibration factor which is independent of the evolution of active regions. It shows only a very weak dependence on the stellar inclination. For example, the inclination dependence can be neglected for inclinations of $45^{\circ}$ and greater; see Fig. 9 from Paper I. Statistically, this corresponds to roughly $70 \%$ of stars.

The model developed in Paper I indicated that the value of $\alpha$ shows a moderate dependence on the ratio between facular and spot areas of the individual active regions at the moment of emergence, $S_{\text {fac }} / S_{\text {spot }}$. This ratio was assumed to be the same for all active regions (see a detailed discussion in Paper I). The dependence of the inflection point position on the facular-tospot area ratio leads to a certain degree of uncertainty (up to $25 \%$ ) when determining stellar rotation periods since the value of $S_{\text {fac }} / S_{\text {spot }}$ for a given star is a priori unknown. At the same time, it allows us to retrieve valuable information about facular versus spot-dominated regimes with regard to the variability of stars where rotation periods can be determined using other methods (see Amazo-Gómez et al. 2020 hereafter, Paper II).

A first test of the gradient of the power spectrum method (hereafter, GPS) was performed in Paper II, where we applied it to solar brightness variations. We showed that in contrast to other methods, GPS allows for an accurate determination of the solar rotation period at all levels of solar activity. Additionally, we analysed time intervals where solar variability was spotdominated and when it was faculae-dominated. We showed that these regimes can be distinguished in the GPS profile due to the substantially different centre-to-limb variations of faculae and spots.

In this study, we apply the GPS method to stars with determined rotation periods from Kepler photometry. The goal here is twofold: firstly, we test the GPS method further before applying it to stars with unknown rotation periods; secondly, we investigate whether the $\alpha$ factor and, consequently, the facular or spot composition of stellar active regions, is dependent on the rotation period. In Sect. 2, we describe the stellar sample we used. In Sect. 3, we present the main results. Our conclusions are summarized in Sect. 4.

\section{Stellar sample selection}

In this study, we consider stars in the field of view (FOV) of the Kepler telescope for which McQuillan et al. (2014) managed to determine rotation periods using the auto-correlation function $(\mathrm{ACF})$. To ensure that the main source of the variability for the selected stars is magnetic activity, we only selected stars on the main-sequence, using $T_{\text {eff }}$ and $\log g$ values from the Huber et al. (2014) catalogue to exclude giants (see Table 1). We note that Huber et al. (2014) calibrated effective temperatures to the infrared flux temperature scale. This resulted in an approximately $200 \mathrm{~K}$ offset from the original Kepler Input Catalogue (KIC) (Pinsonneault et al. 2012). We also precluded stars flagged in the KIC as giant (GS), eclipsing binary (EB), or host stars with planetary transits confirmed (PTC), with planet candidates (PC), and false-positive planets (FP).

We selected two sets of stars with near-solar parameters. The selection criteria for both samples (A and B) are illustrated in Fig. 1 and given in Table 1. Figures 1 and 2 show the variability ranges of the set of selected stars. Kepler observatory provided 4 yr of photometric information, from 2009 to 2013, segmented in 18 quarters $\left(Q_{0}-Q_{17}\right)$ due to the telescope re-orienting itself every 90 days. The Kepler observing quarters resulted in $Q_{0}$ of 10 days, and $Q_{1}$ of 33 days for the commissioning phase and, segmented 90-day LCs for $Q_{2}$ to $Q_{16}$ (see public data release 25, Thompson et al. 2016; Van Cleve \& Caldwell 2016). The second month of $Q_{17}$ was terminated after less than five days of observation, following the failure of reaction wheel 4 .

Sample A (see Fig. 1, panel II) was selected to test the performance of the GPS method for stars with near-solar rotation periods. Hence, in this sample, we considered stars with a narrow range of rotational periods between 24.0 and 27.4 days (i.e. encompassing the sidereal Carrington rotation period of the Sun at 25.4 days) and a broad range of effective temperatures $T_{\text {eff }} \in$ (5000-6000) K. These selection criteria yielded a sample consisting of 686 stars. Among this sample, 282 stars also have 


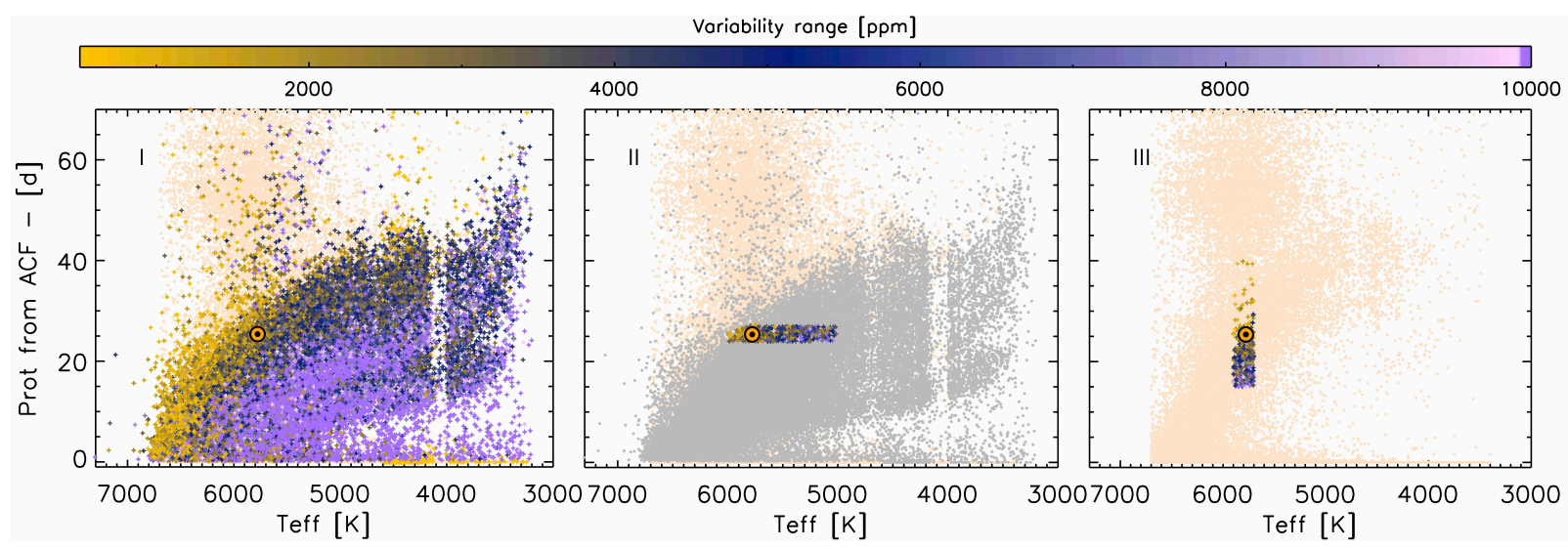

Fig. 1. Panel I: temperature-rotation diagram for a sample of 34030 stars (coloured circles indicating the variability range) with rotation periods determined by McQuillan et al. (2014), along with 55501 stars where the authors found a period but evaluated it as not significant (beige dots; see panel III for a better visualization). Panel II: sample of 34030 stars with determined rotation periods in grey and 55501 stars with non-significant rotation period determinations in beige. For panels II and III, only the stars from sample A (panel II) and sample B (panel III) are shown in colour; see Table 1 for the properties of samples A and B. Panel II illustrates the stellar sample A, selected by near-solar rotation period and temperatures from 5000 to 6000 K. Panel III illustrates stellar sample B, which contains stars with near-solar effective temperature and a broad range of rotation periods. The Sun is represented by the solar symbol $\odot$.

rotation periods obtained by Reinhold \& Gizon (2015) using the generalized Lomb-Scargle periodograms (hereafter, GLS).

Sample B (see Fig. 1, panel III) was selected to study the dependence of the inflection point position on the rotation period. Therefore, in contrast to sample A, we considered stars with a broad range of rotation periods (between 15 and 40 days) but a narrow range of effective temperatures (5700$5900 \mathrm{~K}$, encompassing the solar value of $5778 \mathrm{~K}$ ). These criteria led to the selection of 361 stars for sample B after removing the overlapping targets the with initial sample A. Rotation periods of 172 stars in this sample were also reported in Reinhold \& Gizon (2015).

Between our two samples, we thus considered 1047 Kepler stars in all. The LCs were acquired in the long-cadence mode (i.e. with a cadence of $29.42 \mathrm{~min}$ ). Following McQuillan et al. (2014) and Reinhold \& Gizon (2015), we utilized LCs from $Q_{1}-$ $Q_{14}$ processed with the pre-search conditioning and Bayesian maximum a posteriori approach (PDC-MAP, see Smith et al. 2012). For quarters $Q_{15}-Q_{17}$, only processing with multiscale MAP (PDC-msMAP Stumpe et al. 2014) is available.

In Fig. 2, we plot the distribution of variability ranges in our samples A and B. These variability values are defined by computing the difference between the 95th and 5th percentiles of the sorted flux values for each of the Kepler observing quarters (see Basri et al. 2011) and then taking the median value among the quarters. This defined variability range was chosen versus the approaches based on the standard deviation analysis by Mathur et al. (2014); He et al. (2015) or the smoothed amplitude (10th to 90th) method presented in Douglas et al. (2017), given the higher range of amplitude used in Basri et al. (2011). The selection of the methods mentioned are not expected to compromise the analysed outcome. Additionally, we show solar variability ranges computed using total solar irradiance data (TSI, i.e. total radiative flux from the Sun at 1 A.U.) for 1996-2017 obtained by the Variability of solar IRradiance and Gravity Oscillations (VIRGO; Fröhlich et al. 1997) experiment on the SOlar and Heliospheric Observatory SoHO mission. For these VIRGO data, the entire 1996-2017 observation period was split into 6 Kepler-like time ranges (five 1530-day periods and one 712-day period for a total of 7787-days starting 28 January 1996; see Fig. 3 from Paper II). The solar variability value

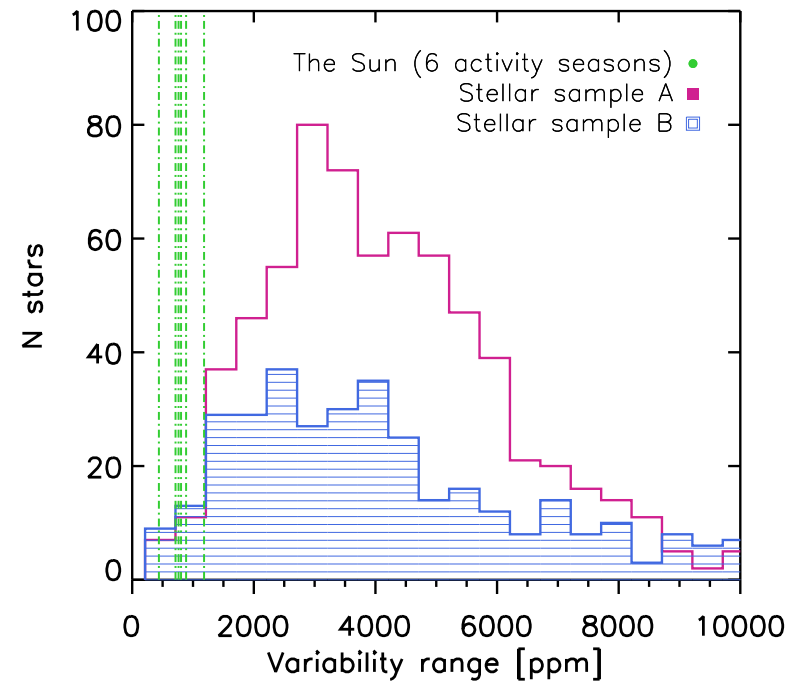

Fig. 2. Histograms for variability ranges from samples A (violet outline) and B (blue rectangles). Vertical green dashed lines represent solar variability calculated for six activity seasons using $21 \mathrm{yr}$ of VIRGO TSI data from Paper II (see text for more details).

for each of the time ranges is represented in Fig. 2 as vertical green dashed lines. This gives a range of solar variability of $\operatorname{Var}_{\odot} \in(400-1300)$ ppm.

Figure 2 shows that most of the stars in our samples are much more variable than the Sun. This agrees with García et al. (2014); Buzasi et al. (2016); Reinhold et al. (2020), who showed that solar-type stars (i.e. stars with near-solar fundamental parameters and rotation periods) are, on average, significantly more variable than the Sun. Furthermore, our samples A and B also contain stars which are cooler and rotate faster than the Sun. These stars are also expected to be more variable than the Sun (see e.g. McQuillan et al. 2014, for a discussion of the dependence of the variability on the rotation period and temperature).

We note that an anomalous low variability of solar-type stars found by Reinhold et al. (2020) does not necessarily imply that the Sun is an outlier. An alternative explanation is that by comparing solar variability to the sample of stars with known 
Table 2. GPS outcome values.

(1)

\begin{tabular}{lcccc} 
KIC & $\begin{array}{c}P_{\text {HFIP }} \\
(\mathrm{d})\end{array}$ & $\begin{array}{c}\sigma P_{\mathrm{HFIP}} \\
(\mathrm{d})\end{array}$ & $\alpha$ & $\sigma \alpha$ \\
\hline 10070928 & 3.78 & 0.108 & 0.173 & 0.0050 \\
10080186 & 3.67 & 0.131 & 0.207 & 0.0074 \\
10080239 & 2.96 & 0.084 & 0.184 & 0.0052 \\
10083970 & 3.08 & 0.097 & 0.188 & 0.0059 \\
10089777 & 3.65 & 0.066 & 0.188 & 0.0034 \\
10091612 & 2.90 & 0.078 & 0.130 & 0.0035 \\
10125510 & 3.78 & 0.180 & 0.161 & 0.0076 \\
10129857 & 4.69 & 0.235 & 0.162 & 0.0081 \\
10136417 & 4.46 & 0.276 & 0.169 & 0.0105 \\
10140949 & 4.12 & 0.127 & 0.182 & 0.0056 \\
10146308 & 3.69 & 0.191 & 0.174 & 0.0090 \\
10064358 & 3.82 & 0.078 & 0.231 & 0.0047
\end{tabular}

(2)

$P_{\text {rot }}$ GPS

rot

19.894

19.894

19.315

15.578

16.210

19.210

15.263

19.894

24.684

23.473

21.684

19.421

20.105
(3)

(4)

4)

Notes. This table contains an example of the GPS outputs, the compared rotation period values from GLS \& ACF, and stellar parameters for 12 randomly selected objects from samples A and B. (1) GPS outcome: in Col. 2, the $P_{\mathrm{HFIP}}$ is given, in Col. 3, its 2-sigma uncertainty, $\sigma P_{\mathrm{HFIP}}$, defined from individual inflection points for each Kepler observing quarter. In Cols. 4 and 5, values of $\alpha$-factor and its 2-sigma uncertainty are reported, respectively. $P_{\text {rot }}$ GPS values in Col. 6, as result of applying Eq. (1) using the factor $\alpha=0.19$. (2) Col. 7 shows the $P_{\text {rot }}$ reported by Reinhold $\&$ Gizon (2015). (3) $P_{\text {rot }}$ and variability values reported by McQuillan et al. (2014) in Col. 8. (4) Cols. 10, 11 and 12 show the log $g$, [Fe/H] and $T_{\text {eff }}$, respectively, taken from Huber et al. (2014). A complete table for the 1047 objects is available at the CDS.

rotation periods, we focus only on a small sub-sample of stars for which the ACF method could return rotation periods (and the Sun most probably would not belong to such a sample). Along this line of reasoning, Reinhold et al. (2020) found that solar levels of photometric variability are typical for stars having near-solar fundamental parameters but unknown rotation periods.

\section{Results and discussion}

In this section, we calculate the position of the inflection point for each star in the samples A and B defined in Sect. 2. Following the methodology described in Papers I and II, we first calculate the power spectra of the stellar brightness variations using a Paul wavelet on the order of six (see Torrence \& Compo 1998) for the Kepler observing quarters $Q_{1}-Q_{17}$. We determined the period corresponding to the high-frequency inflection point, $P_{\mathrm{HFIP}_{\left(\mathrm{Q}_{n}\right)}}$, per quarter and calculate the mean value for $P_{\text {HFIP }}$ over all 17 quarters for each star. This allows us to obtain a unique representative value of $P_{\text {HFIP }}$ per star. The uncertainty is calculated using 2- $\sigma$ of the distribution of the obtained $P_{\mathrm{HFIP}}$ values. Finally, we used the $P_{\text {HFIP }}$ to calculate the stellar rotation period, $P_{\text {rot }}$ (see Table 2 and on-line reference for a compilation of GPS outputs and comparison with GLS and ACF reference values).

In Fig. 3, we plot the mean values of the $P_{\text {HFIP }}$ positions for each of the stars against the rotation periods from Reinhold \& Gizon (2015) (left panel, GLS) and McQuillan et al. (2014) (right panel, ACF). The rotation periods and positions of the inflection points are well-correlated. A linear fit constrained to go through the origin of the coordinate system gives $P_{\mathrm{HFIP}}=0.19 \times P_{\text {rot }}$ with Pearson coefficients for periods of 0.81 from Reinhold \& Gizon (2015) and 0.80 from McQuillan et al. (2014).

The scatter around the linear fits has multiple sources. First, the calibration coefficient between rotation period and inflection point, $\alpha=P_{\mathrm{HFIP}} / P_{\text {rot }}$, depends on the relative roles that bright faculae and dark spots play in generating stellar brightness variations. According to the model presented in Paper I, these roles are regulated by the ratio between facular and spot areas of active regions at the time of emergence, $S_{\text {fac }} / S_{\text {spot }}$ (i.e. zero ratio would lead to a purely spot-dominated star, while very large ratios would correspond to a faculae-dominated star). Secondly, there is an intrinsic statistical uncertainty of the GPS method. For example, in Paper I, we found that even for a star with a fixed $S_{\text {fac }} / S_{\text {spot }}$ ratio, the factor $\alpha$ showed 5-10\% variations from one realization of active regions emergence to another. Finally, there is also an uncertainly in the determination of rotation periods by Reinhold \& Gizon (2015) and McQuillan et al. (2014) (see e.g. Fig. A.2, where we compare the periods from these two sources for the 172 stars of sample B that are common to both).

In Fig. 4, we show calibration factors, $\alpha$, for samples A (top panel) and B (bottom panel). The rotation periods of stars in both samples are taken from McQuillan et al. (2014). In Paper I, we demonstrated that the profile of the high-frequency tail of the power spectrum and, consequently, the values of $\alpha$ depend on the centre-to-limb variations (CLVs) of the brightness contrasts of magnetic features. Since spots and faculae have different CLVs, the value of $\alpha$ depends on their relative contributions to the stellar brightness variations. For the extreme cases, we found that $\alpha$ is about 0.14 for simulated stellar light curves with variability solely determined by faculae and about 0.21 for simulated stars with variability dominated by spots. These values are designated, respectively, by the red and green horizontal dashed lines in Fig. 4. It is reassuring to see that most of the $\alpha$ values for samples A and B appear between these two extreme-cases. Stars with values of $\alpha$ outside of this range (in particular, with $\alpha>0.21$ ) are likely due to: inclination angles below $45^{\circ}$, which can lead to a shift of the inflection point to lower frequencies (see Fig. 9 from Paper I); statistical noise of the GPS method; and possible uncertainties in rotation periods from McQuillan et al. (2014).

For sample A, the ratios are shown as a function of stellar effective temperature from Huber et al. (2014), while for sample $\mathrm{B}$, they are plotted as a function of stellar rotation period from McQuillan et al. (2014). The upper panel of Fig. 4 shows that for near-solar rotation periods (the rotation periods in sample A were constrained between 24 and 27.4 days; see Table 1), the 


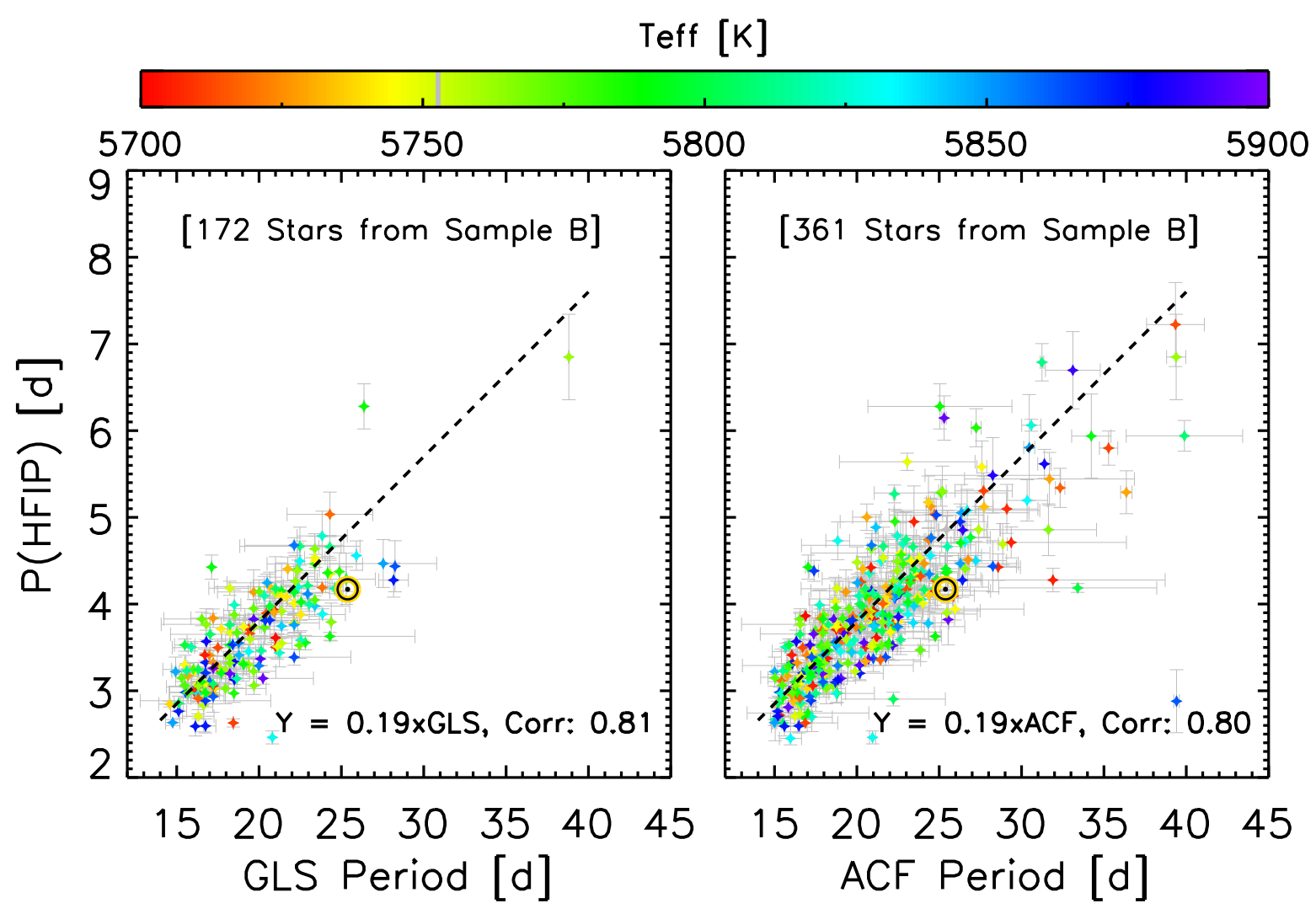

Fig. 3. Position of the high-frequency inflection point $\left(P_{\mathrm{HFIP}}\right)$ plotted against rotation period. Only sample B is shown here. Rotation periods are taken from Reinhold \& Gizon (2015) (left panel) and McQuillan et al. (2014) (right panel). Colours represent the stellar effective temperature, $T_{\text {eff }}$. The Sun is represented by the solar symbol $\odot$. Dashed lines in both panels indicate a linear fit constrained to go through the origin of the coordinate system. A logarithmic visualization is available in the appendix, see Fig. A.1.

position of the inflection point shows no significant dependence on the effective temperature (e.g. the fitting of a slope gives a value of $7.36 \times 10^{-7}$, which is well below the $1 \sigma$ uncertainty of $1.8 \times 10^{-6}$ ). We also note that the mean value of $\alpha=0.19$ is equal to the slope of the regression shown in Fig. 3. This implies that neither the $S_{\text {fac }} / S_{\text {spot }}$ value nor CLVs of facular and spot contrast change significantly within the 5000-6000 K domain of sample A. We note, however, that we cannot conclusively exclude the improbable scenario that the effect from the change of the facular and spot contributions to brightness variability on $\alpha$ is compensated by a change of facular and spot CLVs, such that the net effect on the inflection point is very small.

The bottom panel of Fig. 4 shows that for stars with nearsolar effective temperatures there is a rather weak but statistically significant dependence of the $\alpha$ factor on the rotation period. For example, fitting a linear dependence returns a slope value of $9.3 \times 10^{-4}$ which is 3.8 times larger than its $1 \sigma$ uncertainty of $2.5 \times 10^{-4}$. However, the value of the slope is strongly affected by a couple of slowly rotating stars and, thus, might not represent a trend in the full sample. To better characterize such a trend, we calculated the mean value of the calibration factor in several bins of rotation period values. We compiled the mean $\alpha$ values per several bins of rotation periods; see Table 3 for details. To further illustrate the trend of $\alpha$ values with rotation period, the histogram to the right side of the panel shows the distributions of $\alpha$ values for two rotation periods - one for stars with rotation periods below 21 days and another with rotation periods above 21 days. We can see that the two distributions are clearly shifted relative to each other and the $\alpha$-values of faster rotating stars are larger than those slow-rotating stars.
We note that the " $n$ " number of stars and the amplitude of photometric variability in our samples decreases with rotation period. Consequently, slow rotators might be more affected by photometric noise. We investigated the possible effect of Kepler white noise on the deduced positions of inflection points for the stars in our samples. In Fig. 5, we plot the dependence of the $\alpha$ factor values on the expected Kepler noise levels for each of the stars, calculating the amplitude of the Kepler noise as a function of the Kepler magnitude (following Lammer 2013). The derived precision (called the noise in the context of Kepler) accounts for noise introduced by the instrument and gives it as a function of the Kepler magnitude of the source and the variability of sources (see Fig. A.4).

As much as $99.9 \%$ of the stars in our sample present a Kepler magnitude of 16 mag or fainter. We find that values of the $\alpha$ factor are independent of the Kepler noise, with fits of a linear dependence to samples $\mathrm{A}$ and $\mathrm{B}$ giving slope values well below their $1 \sigma$ uncertainties $\left(7.4 \times 10^{-7}\right.$ and $4.9 \times 10^{-8}$, respectively). Consequently, we do not expect the Kepler noise to affect the positions of inflection points determined for stars in our samples. Furthermore, we note that photometric noise would shift the position of the inflection point to lower frequencies (see Paper II), meaning that it would lead to a trend that is opposite to what we see in the bottom panel of Fig. 4.

A possible explanation of the observed tendency in Fig. 4 is a change in the relative contribution of faculae and spots to stellar rotation variability (or $S_{\text {fac }} / S_{\text {spot }}$ ratio in terms of Paper I) with rotation period. The increase of the $\alpha$ factor with rotation rate implies that the $S_{\text {fac }} / S_{\text {spot }}$ ratio (and, consequently, the contribution of faculae to the rotational variability) is lower in faster 

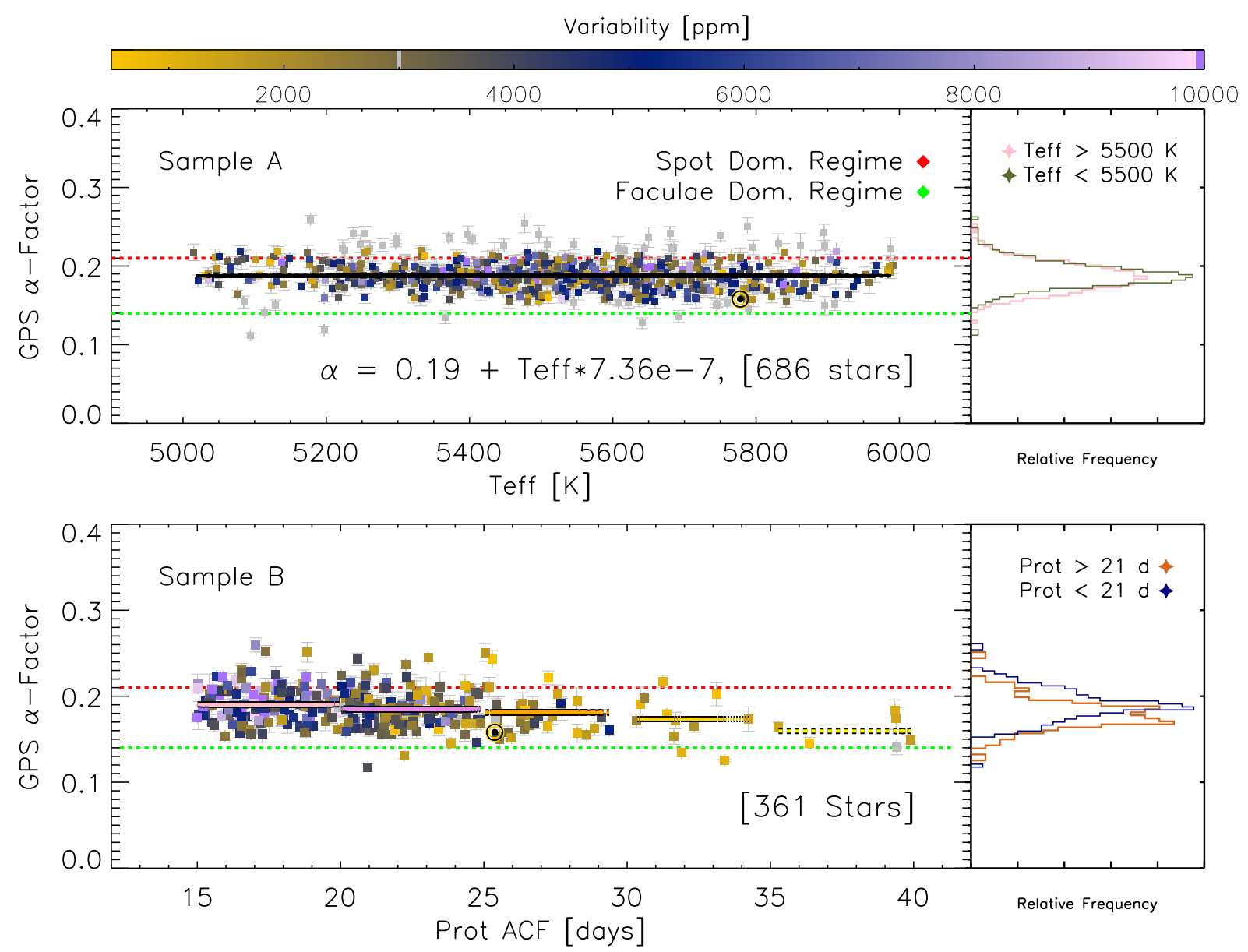

Fig. 4. Top panel: $\alpha$ factor versus $T_{\text {eff }}$ for sample A exhibits consistency across a broad temperature range. The black line corresponds to the linear fit to values with an uncertainty within $2-\sigma$ of the mean of the distribution as shown by coloured squares (grey squares lie outside the 2- $\sigma$ of the distribution). The histograms to the right side of the panel display the distribution of $\alpha$ values for two effective temperature regimes, using $T_{\text {eff }}=5500 \mathrm{~K}$ as a threshold. Bottom panel: $\alpha$ factor versus $P_{\text {rot }}$ from McQuillan et al. (2014) for sample B shows a slight decrease in $\alpha$ with rotation period. The coloured segments indicate the mean of $\alpha$ for the different $P_{\text {rot }}$ ranges as indicated in Table 3. The histograms to the right of the panel indicate the distribution of $\alpha$ values for two rotation period regimens, using $21 \mathrm{~d}$ as a threshold. For both panels, the error bars represent $2-\sigma$ uncertainties of the $\alpha$ values over all Kepler quarters available for each star. The grey squares lie outside of a 2- $\sigma$ of the distribution. The dashed red and green horizontal lines represent the $\alpha$ factor values in the extreme cases with all variability being due to spots $(\alpha=0.21)$ and all due to faculae $(\alpha=0.14)$, respectively ${ }^{1}$.

Table 3. Mean $\alpha$-values in sample B per bin.

\begin{tabular}{lcccccl}
\hline \hline Bin & $n$ & $P_{\text {HFIP }}(\mathrm{d})$ & $\bar{\alpha}$ & $\sigma$ & $\sigma / \sqrt{n}$ & Bin colour \\
\hline 1 & 158 & {$[14-20]$} & 0.190 & 0.001 & $9.0 \times 10^{-5}$ & Pink \\
2 & 148 & {$[20-25]$} & 0.184 & 0.001 & $9.8 \times 10^{-5}$ & Purple \\
3 & 36 & {$[25-30]$} & 0.181 & 0.003 & $5.0 \times 10^{-4}$ & Orange \\
4 & 12 & {$[30-35]$} & 0.173 & 0.006 & $1.9 \times 10^{-3}$ & Gold \\
5 & 6 & {$[35-40]$} & 0.156 & 0.004 & $0.17 \times 10^{-3}$ & Yellow \\
\hline
\end{tabular}

Notes. Compilation of mean $\alpha$-values for $\mathrm{n}$ stars per range of rotation periods, see Fig. 4 .

rotating and, therefore, more active stars. Such a trend is consistent with an extrapolation to higher activities of observed solar behaviour. Indeed, the mean size of spots on the Sun increases during periods of high solar activity (Hathaway 2015; Mandal et al. 2020). At the same time the $S_{\text {fac }} / S_{\text {spot }}$ ratio decreases with the size of active regions and their spot components. An

\footnotetext{
1 A logarithmic visualization of the relation $\alpha$ factor versus $P_{\text {rot }}$ is available in the appendix, see Fig. A.3.
}

extrapolation of these trends to activity levels higher than seen in the Sun results in an increase of the $\alpha$ factor with activity, and, consequently, with rotation rate, as indicated by the bottom panel of Fig. 4.

We note that the ratio $S_{\text {fac }} / S_{\text {spot }}$ between facular and spot areas of the individual magnetic features at the moment of their emergence discussed until now is different from the ratio between "instantaneous" stellar disk coverage by faculae and spots. The former is a property of a magnetic feature during its 


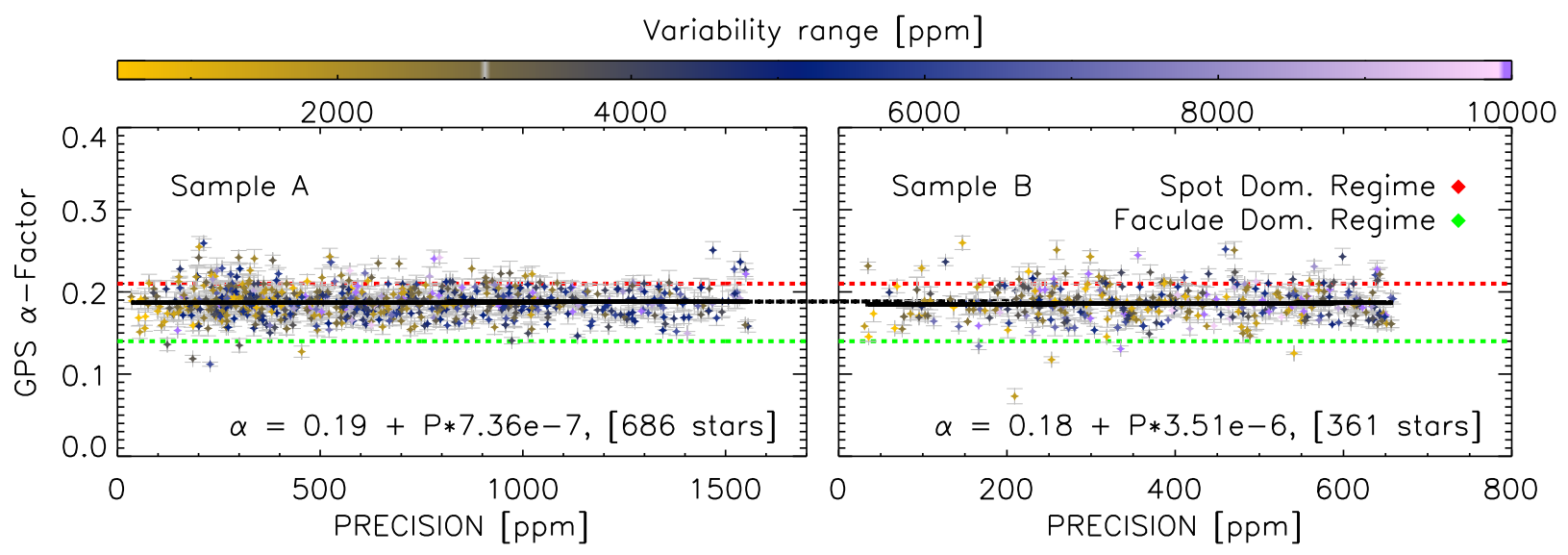

Fig. 5. $\alpha$ factor versus photometric precision for sample A (left panel) and sample B (right panel) for Kepler observations. Those records for single long observation of isolated stars observed in an uncrowded pixel, i.e. light curves of resolved targets without contamination of additional sources. Extreme-case limits for spot- and faculae-dominated stars are shown as horizontal dashed lines in red and green, respectively. The individual data points are coloured according to the detected variability range for that particular star. This is consistent with the preceding figure, Fig. 4 . The error bars represent 2- $\sigma$ uncertainties of the $\alpha$ values distribution over all Kepler quarters available per star. Gray rhomboids represent data points that lie more than $2-\sigma$ from the centre of the distribution.

emergence onto the surface of the star, while the latter is strongly affected by the evolution of the magnetic flux after emergence. For example, in the hypothetical case of facular portions of active regions evolving exactly as spot portions, these two ratio remain the same. In reality, the "instantaneous" ratio is generally significantly larger than that "at the time of emergence" since faculae live longer than spots.

Solar observations show that the ratio between such instantaneous solar-disk coverage by faculae and spots decreases as solar activity increases (Chapman et al. 1997; Foukal 1998). The observed patterns of stellar-brightness variability indicate that this trend also extends to activity values that are significantly higher than those observed on the Sun (Shapiro et al. 2014). Our result indicates that not only the ratio between instantaneous facular and spot disc coverage shows this trend. Also facular to spot area ratio corresponding to individual active regions "at the time of emergence" continues to decrease with increasing level of activity, also beyond the level of solar activity observed until now. We note that this result is not a simple consequence of the drop in the instantaneous ratio. Simulations with a surface flux transport model by Cameron et al. (2010) show that the origin of the decrease in the instantaneous ratio with increasing activity is rather complex. It is, to a great extent, caused by a stronger cancellation of small-scale magnetic field associated with faculae. Consequently, it does not necessarily demand any changes in the structure of the emerging magnetic flux which defines the ratio corresponding to the individual active region at the time of emergence; see the discussion in Paper I.

The bottom panel of Fig. 4 shows that the dependence of $\alpha$ on the rotation period is quite noisy, that is, there is quite a large spread of values for a fixed rotation period. This spread basically covers the entire range of values between faculae- and spot-dominated variability. In particular, it is significantly larger than statistical noise in the inflection point position that we found in Paper I. We speculate that such a large spread implies that the $S_{\text {fac }} / S_{\text {spot }}$ ratio is not uniquely defined by the stellar effective temperature and rotation period.

In Papers I and II, we found that the solar value of the calibration factor $\left(\alpha_{\text {Sun }}=0.158\right)$ is closer to the faculae-dominated case $(\alpha=0.14)$ than to the spot-dominated case $(\alpha=0.21)$. Interestingly, Fig. 4 shows that the solar $\left(\alpha_{\text {Sun }}\right)$ value appears to be rather low relative to that of stars in both of our samples (see also Fig. 3, where the Sun is clearly below the regression line). This is, however, not surprising since most of the stars in our samples are significantly more variable than the Sun even though we selected the stellar sample by the reported detected rotation period and not by the variability (see Fig. 2, cf. Reinhold et al. 2020). This implies that these stars are also more active than the Sun (see also Zhang et al. 2020, who showed that stars with known near-solar rotation periods have systematically higher values of S-index than the Sun). Therefore, we can expect that their $S_{\text {fac }} / S_{\text {spot }}$ ratios are smaller and, consequently, their $\alpha$ factors are larger.

\section{Summary}

In this study, we developed the GPS method, which is a novel means for determining stellar rotation periods from photometric time series. Instead of basing this determination on the more traditional means of identifying the strongest peak in a LombScargle periodogram or a maximum of the auto-correlation function, we identify the steepest point (i.e. the inflection point) in the global wavelet power spectrum of stellar brightness variations. In Paper II, we showed that while the solar brightness contributions from faculae and spots can oppose each other to reduce any peak due to the rotation period from Lomb-Scargle periodograms and auto-correlation functions, it has only a very minor effect on the location of this high-frequency inflection point and the resulting ratio between the period corresponding to the inflection point and the actual rotation period, $\alpha$. In Paper I, the factor of $\alpha$, however, shows a moderate dependence on the relative contribution of faculae to stellar brightness variations. Therefore, identifying the position of the inflection point allowed determination of the rotation period in stars where other methods fail (with an internal uncertainty of about $25 \%$ ). At the same time, this GPS method allows us to assess the relative role of faculae in stars with known rotation periods. In Paper II, we tested the performance of the GPS method against solar photometric data. We demonstrated that in contrast to other methods, the GPS method allows for an accurate determination of the solar rotation period independently of the solar activity level. 
In this study, we applied the GPS method to 1047 F-, G- and K-type stars with rotation periods as reported in McQuillan et al. (2014). We show that the position of the high-frequency inflection point is well-correlated with the rotation periods of stars in the two samples we analysed, providing further validation of the GPS method. We emphasize that the stellar light curves analysed in this study and the solar light curves analysed in Paper II are quite different: the amplitudes of brightness variability in the stellar samples in this study are generally higher than that of the Sun, and the stellar brightness modulation are much more regular over rotational timescales.

We find that the $\alpha$ factor increases with rotation rate, indicating that faculae become less important on stars rotating faster than the Sun. We have also found that the facular contribution to solar brightness variability is larger than its contribution to brightness variability in a sample of stars having near-solar rotation periods and temperatures. We attribute this to a selection effect since the rotation periods of stars with brightnessvariability patterns similar to that of the Sun are rather difficult to measure via the ACF method for rotation-period determinations and, thus, there is a dearth of such stars in our sample. Consequently, our results indicate that in addition to being more active than the Sun (see also Reinhold et al. 2020; Zhang et al. 2020), the stars with near-solar effective temperatures and nearsolar rotation periods determined by McQuillan et al. (2014) have different compositions of active regions (with smaller facular contributions). The GPS method for determining rotation periods could thus prove to be an important contributor to enhance the lower-than-expected number of G-type stars with near-solar rotation periods reported by van Saders et al. (2019). This method can also improve the solar-stellar comparison as in Reinhold et al. (2020). The outcome of GPS might bring a new perspective in understanding stellar activity.

While we focus in this study on applying GPS to stars with known rotation periods, in a forthcoming study, we plan to apply the GPS method to Kepler stars with previously unknown rotation periods as well as to TESS stars. This might help establish a new and more complete sample of stars having near-solar rotation periods, based upon which we can investigate whether solar variability still appears anomalously low in comparison to stars in this broader sample.

This will be of importance to the exoplanet community, since the knowledge of rotation periods will help identify radial velocity jitter from planetary signals (see Oshagh 2018; Faria et al. 2020; Hojjatpanah et al. 2020). The anticipated GPS-determined expanded database of stellar rotation periods could also bring crucial information for ongoing and upcoming surveys such as NIRPS-HARPS and ESPRESSO (see Pepe et al. 2010; Bouchy \& Doyon 2018). Additionally, the precise determination of host-star rotation periods is important for recovering accurate exoplanet radii, which will be crucial for future searches of transiting Earths or super-Earths in the light curves of solar twins in the PLATO field (see Rauer et al. 2014).

Acknowledgements. We would like to thank the referee for the constructive comments which helped to improve the quality of this paper. This work was supported by the International Max-Planck Research School (IMPRS) for Solar System Science at the University of Göttingen and European Research Council under the European Union Horizon 2020 research and innovation program (grant agreement by the No. 715947). M.O. acknowledges the support of the Deutsche Forschungsgemeinschft (DFG) priority program SPP 1992, Exploring the Diversity of Extrasolar Planets (RE 1664/17-1). E.M.A.G. and M.O. also acknowledge the support of the FCT/DAAD bilateral grant 2019 (DAAD ID: 57453096).
Financial support was also provided by the Brain Korea 21 plus program through the National Research Foundation funded by the Ministry of Education of Korea and by the German Federal Ministry of Education and Research under project 01LG1209A. We would like to thank the International Space Science Institute, Bern, for their support of science team 446 and the resulting helpful discussions. This paper includes data collected by the Kepler mission. Funding for the Kepler mission is provided by the NASA Science Mission directorate. The data presented were obtained from the Mikulski Archive for Space Telescopes (MAST). STScI is operated by the Association of Universities for Research in Astronomy, Inc., under NASA contract NAS5-26555.

\section{References}

Aigrain, S., Llama, J., Ceillier, T., et al. 2015, MNRAS, 450, 3211

Amazo-Gómez, E. M., Shapiro, A. I., Solanki, S. K., et al. 2020, A\&A, 636, A69 Angus, R., Morton, T., Aigrain, S., Foreman-Mackey, D., \& Rajpaul, V. 2018, MNRAS, 474, 2094

Basri, G. 2018, ApJ, 865, 142

Basri, G., Walkowicz, L. M., Batalha, N., et al. 2011, AJ, 141, 20

Borucki, W. J., Koch, D., Basri, G., et al. 2010, Science, 327, 977

Bouchy, F., \& Doyon, R. 2018, in European Planetary Science Congress, EPSC2018-1147

Buzasi, D., Lezcano, A., \& Preston, H. L. 2016, J. Space Weather Space Climate, 6, A38

Cameron, R. H., Jiang, J., Schmitt, D., \& Schüssler, M. 2010, ApJ, 719, 264

Chapman, G. A., Cookson, A. M., \& Dobias, J. J. 1997, ApJ, 482, 541

Douglas, S. T., Agüeros, M. A., Covey, K. R., \& Kraus, A. 2017, ApJ, 842, 83

Faria, J. P., Adibekyan, V., Amazo-Gómez, E. M., et al. 2020, A\&A, 635, A13

Foukal, P. 1998, ApJ, 500, 958

Fröhlich, C., Crommelynck, D. A., Wehrli, C., et al. 1997, Sol. Phys., 175, 267

García, R. A., Ceillier, T., Salabert, D., et al. 2014, A\&A, 572, A34

Hathaway, D. H. 2015, Liv. Rev. Sol. Phys., 12, 4

He, H., Wang, H., \& Yun, D. 2015, ApJS, 221, 18

Hojjatpanah, S., Oshagh, M., Figueira, P., et al. 2020, A\&A, 639, A35

Huber, D., Silva Aguirre, V., Matthews, J. M., et al. 2014, ApJS, 211, 2

Lammer, H. 2013, Origin and Evolution of Planetary Atmospheres (Springer,

Berlin Heidelberg), http : //doi . org/10.1007/978-3-642-32087-3

Lanza, A. F., \& Shkolnik, E. L. 2014, MNRAS, 443, 1451

Mandal, S., Krivova, N. A., Solanki, S. K., Sinha, N., \& Banerjee, D. 2020, A\&A, 640, A78

Mathur, S., Salabert, D., García, R. A., \& Ceillier, T. 2014, J. Space Weather Space Climate, 4, A15

McQuillan, A., Mazeh, T., \& Aigrain, S. 2014, ApJS, 211, 24

Nielsen, M. B., Gizon, L., Schunker, H., \& Karoff, C. 2013, A\&A, 557, L10

Oshagh, M. 2018, Asteroseismology and Exoplanets: Listening to the Stars and Searching for New Worlds, 49, 239

Pepe, F. A., Cristiani, S., Rebolo Lopez, R., et al. 2010, in Proc. SPIE, Vol. 7735, Ground-based and Airborne Instrumentation for Astronomy III, 77350F

Pinsonneault, M. H., An, D., Molenda-Żakowicz, J., et al. 2012, ApJS, 199, 30

Rauer, H., Catala, C., Aerts, C., et al. 2014, Exp. Astron., 38, 249

Reinhold, T., \& Gizon, L. 2015, A\&A, 583, A65

Reinhold, T., Shapiro, A. I., Solanki, S. K., et al. 2020, Science, 368, 518

Santos, A. R. G., García, R. A., Mathur, S., et al. 2019, ApJS, 244, 21

Shapiro, A. I., Solanki, S. K., Krivova, N. A., et al. 2014, A\&A, 569, A38

Shapiro, A. I., Solanki, S. K., Krivova, N. A., et al. 2017, Nat. Astron., 1, 612

Shapiro, A. I., Amazo-Gómez, E. M., Krivova, N. A., \& Solanki, S. K. 2020, A\&A, 633, A32

Smith, J. C., Stumpe, M. C., Van Cleve, J. E., et al. 2012, PASP, 124, 1000

Solanki, S. K. 1993, Space Sci. Rev., 63, 1

Solanki, S. K., Inhester, B., \& Schüssler, M. 2006, Rep. Progr. Phys., 69, 563

Stumpe, M. C., Smith, J. C., Catanzarite, J. H., et al. 2014, PASP, 126, 100

Thompson, S. E., Caldwell, D. A., Jenkins, J. M., et al. 2016, Kepler Data Release 25 Notes, Kepler Science Document

Torrence, C., \& Compo, G. P. 1998, Bull. Am. Meteorol. Soc., 79, 61

Van Cleve, J. E., \& Caldwell, D. A. 2016, Kepler Instrument Handbook, Tech. rep.

van Saders, J. L., Pinsonneault, M. H., \& Barbieri, M. 2019, ApJ, 872, 128

Walkowicz, L. M., \& Basri, G. S. 2013, MNRAS, 436, 1883

Witzke, V., Reinhold, T., Shapiro, A. I., Krivova, N. A., \& Solanki, S. K. 2020, A\&A, 634, L9

Zhang, J., Shapiro, A. I., Bi, S., et al. 2020, ApJ, 894, L11 
Appendix A: Additional figures

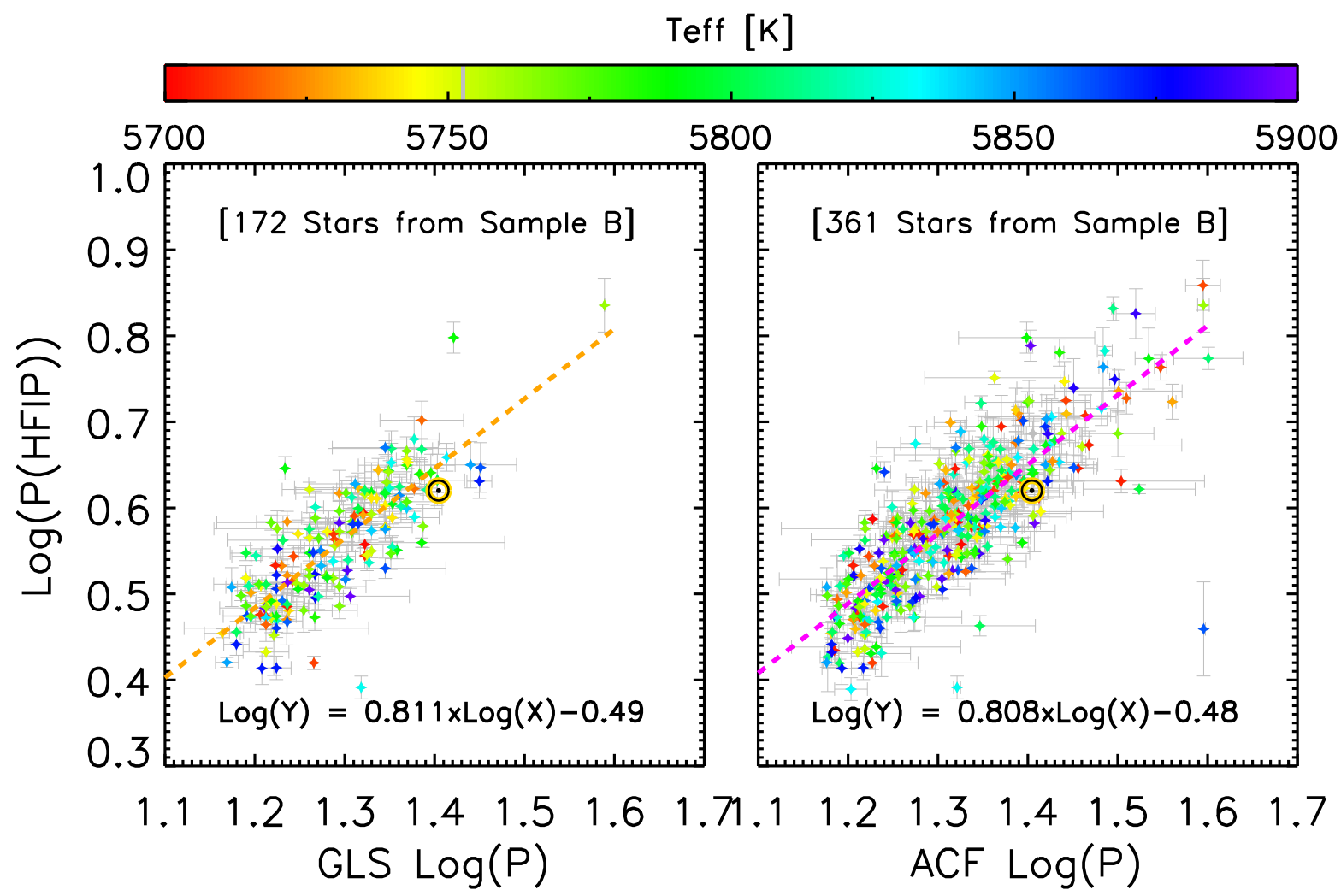

Fig. A.1. Logarithmic visualisation of Fig. 3.

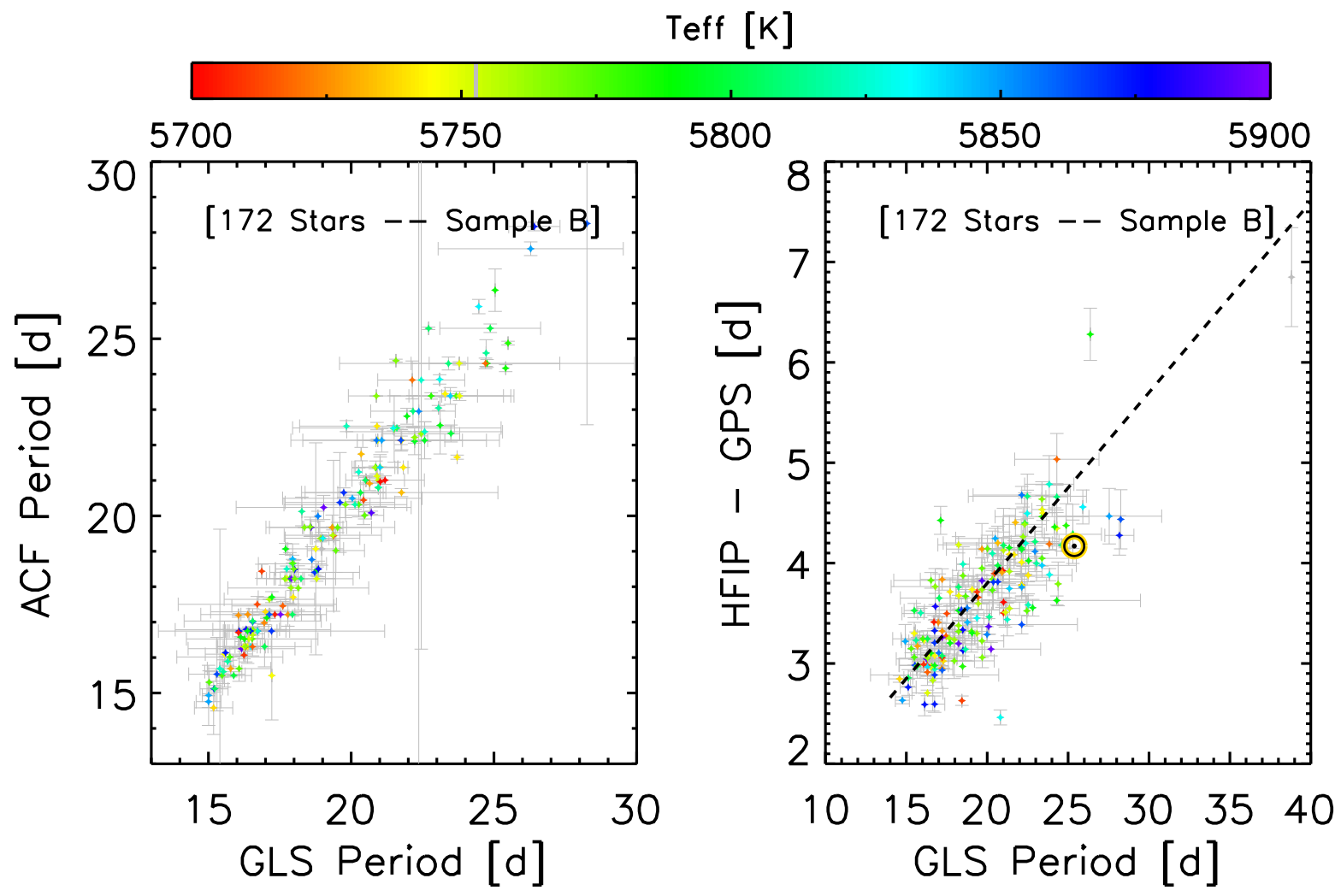

Fig. A.2. Comparison for the 172 stars in sample B with reported rotation periods by the ACF, GLS, and GPS methods. Left: ACF versus GLS. Right: HFIP-GPS versus GLS. Scatter colour to visualise the temperature. The comparison is made in a similar range scale for a better visualisation. 


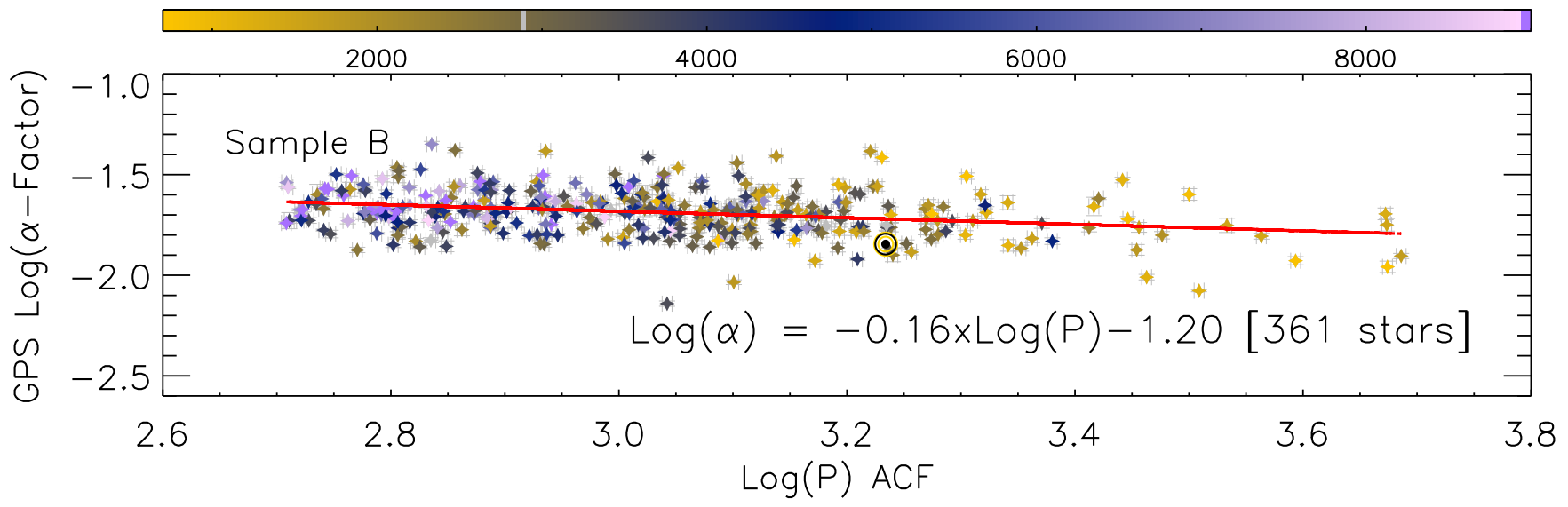

Fig. A.3. Logarithmic visualisation of bottom panel in Fig. 4.

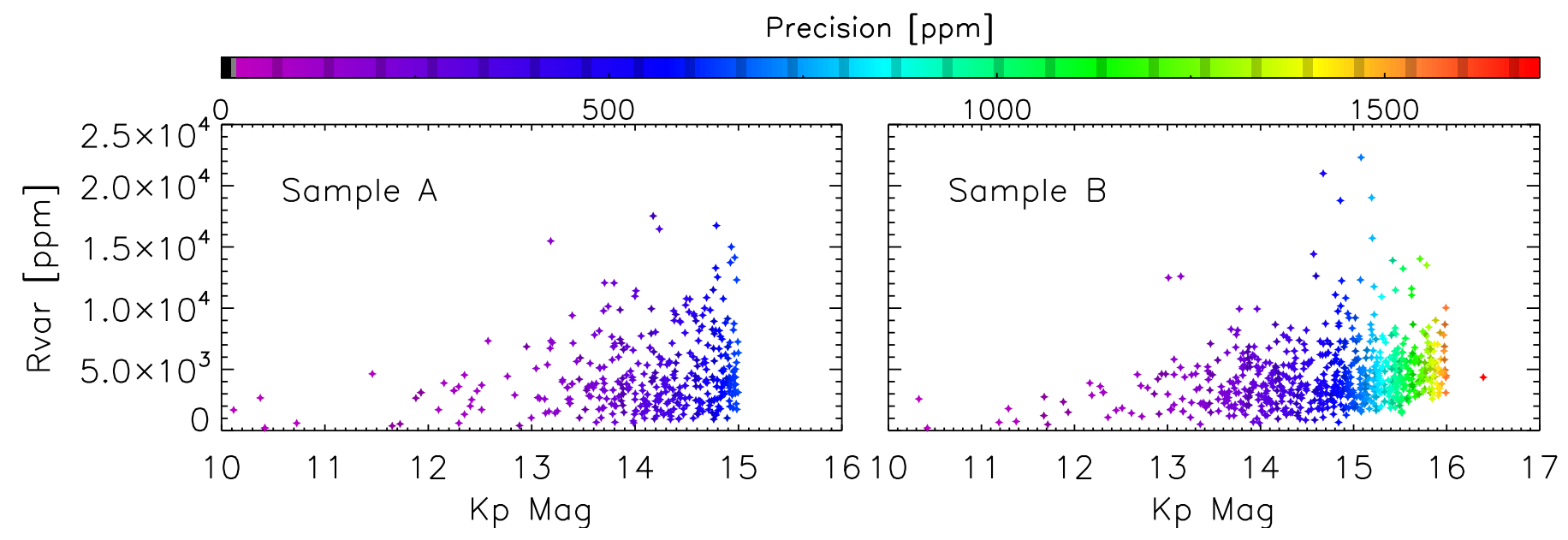

Fig. A.4. Variability range in ppm versus Kepler magnitude Kmag. The colour bar indicates Kepler precision. 